\title{
Programa de detección del cáncer cervicouterino: políticas públicas y experiencias de los actores que implementan el programa en el estado de Veracruz, México
}

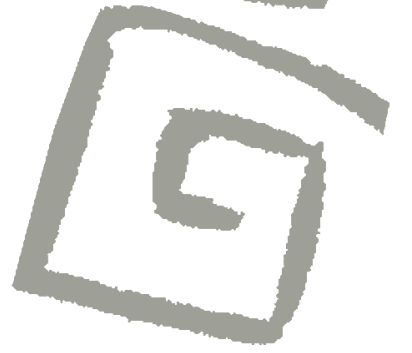

\author{
Screening program for cervical cancer: public policies \\ and experiences of actors who implement the program \\ in the state of Veracruz, Mexico
}

\section{Zuanilda Mendoza González}

${ }^{1}$ Doctora en Antropología Social. Profesora de carrera Departamento de Historia y Filosofía de la Medicina, Universidad Nacional Autónoma de México México. $\bowtie$
RESUMEN El propósito fue analizar cómo se desarrolla el Programa de Detección del Cáncer Cervicouterino en una clínica de displasias y algunos centros de salud en el estado de Veracruz, a través de las representaciones y prácticas de los actores sociales que implementan el programa. Para ello, se realizaron entrevistas en profundidad y observación de la práctica de estos prestadores de servicios durante varios periodos a lo largo de 3 años entre 2009 y 2011. A partir de la información obtenida pretendemos dar cuenta de las dificultades, aciertos y resultados que dicho programa tiene como parte de una política pública, sobre la base de que las políticas en salud tienen como prioridad que toda la población se vea beneficiada por los servicios de atención preventiva y curativa, y si bien la evidencia nos muestra que las poblaciones marginadas no se ven beneficiadas por dichos programas, pareciera que esta información no permea los saberes populares y médicos.

PALABRAS CLAVES Programas de Prevención de Cáncer de Cuello del Útero; México.

\begin{abstract}
The aim of this article is to analyze the way in which the Screening Program for Cervical Cancer is carried out in a dysplasia clinic and related health centers in the state of Veracruz, through the representations and practices of the social actors who implement the program. In order to do so, in-depth interviews and observations of the practices of health service providers were carried out during different periods over the course of three years, from 2009 to 2011. Through the information obtained, the article explores the difficulties, achievements and results of this program as part of a public policy. Although a priority of public health policy is to see the whole population benefit from preventive and curative health care services, evidence shows that marginalized populations are not benefitted by such programs; such information does not however seem to permeate popular and medical knowledge.
\end{abstract}

KEY WORDS Cervix Neoplasms Prevention Programs; Health Policy; Mexico. 


\section{INTRODUCCIÓN}

El cáncer cervicouterino sigue representando un problema de salud pública en México, por su magnitud y trascendencia en las esferas individual, familiar, social y económica. Esta enfermedad es motivo de dolor físico y sufrimiento para las mujeres enfermas y sus familiares, y los costos del diagnóstico, tratamiento y control se ubican entre los más altos en la medicina, motivo por lo que se le consideran de tipo catastrófico para el individuo y las instituciones ${ }^{(1)}$.

Se trata de una neoplasia de relevancia por la magnitud que presenta respecto a la morbilidad y mortalidad, sobre todo si se toma en cuenta su alto potencial de ser prevenible a fin de evitar la mortalidad por esta causa. Es una enfermedad multifactorial y de lenta progresión, que se desarrolla asociada a la infección persistente por algunos de los tipos del virus del papiloma humano (VPH) identificados como de alto riesgo, que presenta en forma gradual lesiones de bajo o alto grado (displasia) y, en ausencia de tratamiento, evolucionan hacia el cáncer. Identificar que la infección por VPH es la principal causa asociada a la presencia de este cáncer, dirige el esfuerzo institucional hacia acciones preventivas para lograr un menor riesgo de contagio, sin dejar de reconocer que los factores sociales y reproductivos influyen de manera preponderante en una mayor probabilidad de infección por $\mathrm{VPH}^{(2)}$.

La falta de un diagnóstico oportuno se ha atribuido como principal causa de mortalidad, condicionada por factores como las barreras de accesibilidad a la atención médica, los aspectos sociales y culturales que impiden la detección y la solicitud de demanda a los servicios ante los primeros síntomas de la enfermedad. Por lo tanto, se reconoce que los servicios de salud deben fortalecer las acciones dirigidas a la educación para la salud, promover la prevención primaria por inmunización y la promoción secundaria a través de la detección oportuna, así como contar con los recursos de atención médica necesarios para atender la demanda en los casos nuevos identificados que contribuyan a disminuir la mortalidad por esta causa ${ }^{(3)}$.

La prioridad de las políticas en salud es que toda la población, cualquiera sea su condición socioeconómica y el grupo étnico al que pertenezca, tenga las mismas oportunidades y calidad en la accesibilidad y servicios de atención preventiva y curativa. La evidencia muestra que poblaciones marginadas, en términos sociales, geográficos y económicos, tienen una mayor probabilidad de morir por cánceres prevenibles, lo cual se puede atribuir, entre otros factores, a que no reciben un diagnóstico oportuno y, por tanto, el tratamiento es tardío ${ }^{(4,5)}$.

En este trabajo, nuestro interés es dar cuenta de algunos aspectos sobre cómo se desarrolla el Programa de Detección del Cáncer Cervicouterino en una clínica de displasia y algunos centros de salud en el estado de Veracruz, enfocándonos en las dificultades, aciertos y resultados que dicho programa tiene como parte de una política pública: ¿cuál es la opinión de los actores sociales que implementan el programa de detección en cáncer cervicouterino en el estado?, ¿cuáles han sido sus limitaciones, problemas y resultados?, ¿y cómo se apropian o no los actores sociales involucrados? La información forma parte de una investigación mayor titulada "Saberes acerca del cáncer cervicouterino de profesionales y pacientes en una clínica de displasia del estado de Veracruz".

\section{El cáncer cervicouterino desde los datos}

Los datos epidemiológicos nos muestran la relación que guarda este tipo de cáncer con condiciones de desigualdad social. El cáncer cervicouterino es la cuarta neoplasia en frecuencia entre las mujeres, a nivel mundial, el $85 \%$ de estos cánceres se dan entre los países en vías de desarrollo, con fuertes diferencias entre tasas de 43,2 por 100.000 mujeres en África Oriental, a tasas de 5,4 por 100.000 mujeres en Asia Oriental, la tendencia de la mortalidad es descendente al disminuir su incidencia, presentar mejores condiciones 
estructurales y una respuesta más eficiente de los servicios de salud( ${ }^{(6)}$.

En América Latina es la segunda neoplasia más común en mujeres. La mortalidad en la región es de 8,7 defunciones por 100.000 mujeres, y el $75 \%$ ocurre en seis países: Brasil, México, Colombia, Perú, Venezuela y Argentina. Sin embargo, la mortalidad es más alta en Guyana con 21,9, Bolivia con 21,0 y Nicaragua con $18,3^{(7,8)}$.

En México, es la segunda causa de muerte por cáncer en la mujer, con una incidencia de 23,3 casos por 100.000 mujeres. En el año 2012, se registraron 3.832 defunciones en mujeres con una tasa cruda de 6,4 defunciones por 100.000 mujeres. En el grupo específico de mujeres de 25 años y más, la tasa cruda es de 11,8 defunciones por 100.000 mujeres y un promedio de edad a la defunción de 59,03 años. Las entidades con mayor mortalidad por cáncer del cuello uterino son Colima, Oaxaca, Veracruz, Morelos y Chiapas. Por el contrario, solo el $42 \%$ de las defunciones por cáncer de cuello uterino se registraron en mujeres con derechohabiencia, y alcanzaron una tasa de 14 por 100.000 entre las mujeres de 25 años y más sin derechohabiencia ${ }^{(9,10)}$.

En México, la mortalidad por cáncer de cuello uterino disminuyó un $37,6 \%$, durante el periodo 2000-2012, al pasar de 4.585 a 3.832 defunciones. Durante este periodo se planteó la meta de disminuir la tasa de mortalidad nacional por cáncer de cuello uterino, lo cual no se consiguió al mantenerla un punto por arriba de lo planteado, lo que mantiene a México como el país con la mortalidad más alta por cáncer de cuello uterino dentro de los países de la Organización para la Cooperación y Desarrollo Económicos (OCDE) ${ }^{(4)}$.

\section{Política pública en salud y cáncer cervicouterino}

En 1974 se estableció en México el Programa Nacional de Detección de Cáncer Cervicouterino y, desde 1992, se ha observado una disminución discreta pero sostenida de la mortalidad por esta enferme$\operatorname{dad}^{(11)}$. México cuenta con una norma oficial
(NOM-014-SSA2-1994) "Sobre el control y tratamiento del cáncer cérvico-uterino" que define los lineamientos a seguir, tanto en el programa nacional de tamizaje, como en el tratamiento de los casos identificados ${ }^{(12)}$.

Es importante mencionar que las pruebas de detección ofrecen la mejor oportunidad para reconocer el cáncer de cuello uterino en una etapa temprana, cuando el tratamiento puede ser eficaz. Además, pueden prevenir la mayoría de los cánceres cervicales, mediante la detección de los cambios anormales de las células del cuello uterino, para que puedan ser tratados antes de que se conviertan en un cáncer, por ser uno de los cánceres que se puede tratar con más éxito, si se detecta a tiempo.

En México, se proporciona atención médica aproximadamente a 9.000 mujeres con cáncer cervicouterino invasor y se registran 4.000 muertes anualmente ${ }^{(9)}$. En los países desarrollados se ha observado una disminución de la mortalidad por esta causa, que se asocia con el porcentaje de población incluida en los programas de tamizaje. En EE.UU., la tasa de mortalidad ha disminuido más del 50\% durante los últimos 30 años relacionada con la eficacia de las pruebas de Papanicolaou ${ }^{(13)}$.

A pesar de los beneficios reconocidos de las pruebas de detección, no todas las mujeres pueden aprovecharlos. La mayoría de estos casos de cáncer se encuentran en mujeres que nunca se han sometido a una prueba de Papanicolaou o que no se la han hecho recientemente. Las mujeres sin acceso a los servicios de salud o que viven en condiciones de pobreza tienen menos probabilidad de acceder a las pruebas de detección en el momento indicado ${ }^{(4,5)}$.

En los países en desarrollo, los programas de tamizaje basados en la citología no han tenido el éxito deseado, en la detección temprana del cáncer cervicouterino, debido a que se requieren múltiples recursos para realizar cada uno de los pasos del programa y no siempre se cuenta con la infraestructura necesaria ${ }^{(11)}$.

La infraestructura de servicios para el tamizaje y diagnóstico de dichas neoplasias se caracteriza por su complejidad y alto costo. 
No obstante, en nuestro país se han presentado avances en la ampliación de la infraestructura del sector salud para el manejo de cáncer de la mujer. Para el caso de la detección y atención de lesiones precursoras es necesario contar con clínicas de colposcopías y laboratorios de citología, patología y biología molecular, además de recursos humanos capacitados. Dichos recursos humanos han crecido durante los últimos años en el sector salud, pero su distribución a nivel institucional no es equitativa. No obstante, el crecimiento es insuficiente para satisfacer la cada vez mayor demanda que exige la ampliación de la infraestructura material. Surge la necesidad de la creación de nuevos especialistas y el fortalecimiento técnico de los existentes. Esto es más evidente en la atención colposcópica: las 428 clínicas se distribuyen de manera desigual en las entidades e instituciones, haciendo necesaria una mayor vinculación entre $s^{(4,14)}$.

Las estrategias implementadas para el manejo del cáncer cervicouterino en el Sistema Nacional de Salud mexicano son: la elaboración de materiales educativos, publicidad educativa, la implementación de la vacunación contra la infección por VPH iniciada el 2008 en los 125 municipios con menor índice de desarrollo humano, en adolescentes de 12 a 16 años, de 2009 a 2012 se extendió a más municipios del país hasta finalmente integrarse al esquema nacional de vacunación, adquiriendo carácter universal para las niñas que cursan el $5^{\circ}$ año de primaria, así como para las no escolarizadas de 11 años, la introducción de la detección biomolecular de los serotipos de VPH asociados a cáncer de cuello uterino en mujeres de 35 a 64 años de edad y la consolidación del Sistema de Información en Cáncer de la Mujer (SICAM) para el monitoreo, evaluación y vigilancia epidemiológica del cáncer de la mujer en la Secretaría de Salud.

Durante el periodo de 2000-2012, se incrementó la cobertura de la prueba de Papanicolaou en mujeres de 25 a 64 años, al pasar de $26,2 \%$ en el año 2000 a 45,5\% en 2012. La cobertura de detección en los últimos 12 meses, incluyendo la prueba de Papanicolaou o la prueba del VPH, fue de $50,1 \%$ en las mujeres entrevistadas en 2012, muy por debajo de el parámetro internacional del $80 \%$. También se reporta una cobertura diferencial entre mujeres con o sin derechohabiencia $(72,3 \%)$ y aquellas sin seguridad social $(49,9 \%)$, y con variaciones amplias entre entidades, con su valor más bajo en Oaxaca $(36,4 \%)$ y más alto en Campeche $(54,1 \%)^{(15)}$.

\section{La detección del cáncer cervicouterino: sus dificultades}

La Organización Mundial de la Salud (OMS) reconoce dos componentes en los programas de detección temprana del cáncer: el tamizaje y el diagnóstico temprano de la enfermedad. Ambos componentes dependen no solo de la capacidad técnica y científica para la realización del programa, sino de forma muy importante de la aceptación de la población de las acciones programadas y de la capacitación del personal para hacer de esta búsqueda una acción sistemática en su práctica diaria ${ }^{(16)}$.

En México, el programa de detección oportuna del cáncer cervicouterino ha operado por más de tres décadas sin que ello se haya reflejado en la reducción esperada de la mortalidad por esta causa en la población femenina del país. La ausencia de efecto del programa es contraria a la experiencia internacional. En la Unión Europea, el programa redujo hasta un $70 \%$ los años de vida perdidos prematuramente entre las mujeres de 25 a 65 años de edad.

En el caso de México, diversos estudios han identificado las áreas de ineficiencia y las acciones correctivas necesarias en el programa, que son: la ampliación de la meta de citologías, la toma de muestras con periodicidad trianual en mujeres con resultados negativos, la elevación de la calidad de las muestras y su interpretación, la capacitación para la toma de muestras y el readiestramiento para su lectura, la notificación personalizada a los casos positivos, el fortalecimiento del programa de divulgación y el establecimiento de un sistema de vigilancia epidemiológica, así 
como la garantía de tratamiento en mujeres con anormalidades detectadas.

Las modificaciones al programa buscan que se ajuste a los estándares internacionales del $10 \%$ de error en la calidad del espécimen citológico, y del $15 \%$ en el diagnóstico tanto de falsos negativos como de falsos positivos. En la evaluación realizada al programa se estimó que más de la mitad de las muestras (64\%) son de baja calidad, y en más de la tercera parte existe imprecisión diagnóstica, que incluye el informe de falsos negativos (del $10 \%$ al $54 \%$ ) y de falsos positivos (del $0 \%$ al $52 \%$ ). Una quinta parte de las usuarias no llegan a conocer los resultados del estudio completo (del $18,5 \%$ al $22,2 \%)^{(11,17)}$. Los diagnósticos erróneos o la ausencia de tratamiento en los casos detectados positivos representan un fracaso para el sector y una tragedia en la vida de estas mujeres.

En décadas recientes se han desarrollado nuevas intervenciones para fortalecer la prevención contra el cáncer cervicouterino. Entre ellas figuran el desarrollo de la vacuna contra los dos tipos más frecuentes del VPH de alto riesgo y la detección de infecciones por el VPH mediante la captura de híbridos $(\mathrm{CH})$. Puesto que la vacuna solo confiere inmunidad para los tipos de VPH precursores del $70 \%$ de los casos de cáncer cervicouterino, aun con cobertura universal, resulta necesario continuar con el tamizaje para la identificación de los casos sin protección inmunológica por vacunación.

Cada año se realiza un promedio de 7,2 millones de tamices anuales mediante citología cervical (Papanicolaou) de forma gratuita, de los cuales una fracción de 1,2\% resulta positiva; sin embargo la cobertura aun no alcanza al $80 \%$ de la población nacional y es diferencial por estados, siendo los estados del sur los que muestran una cobertura más baja (menor al $49 \%)^{(3)}$.

Pese a los logros alcanzados en el Programa Nacional de Tamizaje del Cáncer Cervicouterino se presentan problemas operativos, entre ellos, la baja sensibilidad del $\mathrm{Pa}$ panicolaou, de tal manera que casi en el $40 \%$ de los casos, en su mayoría en etapas tempranas, no se reconoce; se requiere personal y equipo especializado para asegurar una buena toma y la interpretación de la prueba depende de la capacidad del cito tecnólogo. Por último, los esfuerzos para aumentar la cobertura del programa de tamizaje han arrojado pocos resultados debido a barreras de acceso por cuestiones geográficas y situaciones de índole cultural ${ }^{(17,18)}$.

Pese a la implementación del programa se reconocen problemas operativos, por lo que nuestra intensión en este texto es partir de la experiencia de los sujetos involucrados en las acciones y que hacen reales las políticas públicas en salud.

\section{El Programa Nacional de Tamizaje del Cáncer Cervicouterino en el estado de Veracruz}

El programa se realiza en el estado de Veracruz desde la década de 1980, pero a partir del año 2010 sufre modificaciones por indicaciones de la Subsecretaria de Prevención y Promoción de la Salud y el Centro Nacional de Equidad de Género y Salud Reproductiva, modificaciones que parten de los cuestionamientos a la implementación del Programa:

- Elevadas tasas de mortalidad por cáncer cervicouterino.

- Baja cobertura en áreas rurales y urbano marginadas.

- Deficiente obtención de la muestra de Papanicolaou.

- Elevado índice de resultados falsos negativos de Papanicolaou.

- Fallas en el seguimiento de la paciente.

- Insuficiente control de calidad en colposcopía con sobretratamiento.

Veracruz fue uno de los estados en los que se implementó de forma piloto la introducción de la prueba de ADN de VPH, cuya fundamentación es la mayor sensibilidad para identificar a pacientes con lesiones de alto riesgo, a través de una combinación de citología y tipificación del VPH. Se recurrió a la selección de zonas de alto riesgo y marginación, realizadas a través de caravanas 
para la toma de la prueba de tipificación de VPH y cuando era posible de autotoma vaginal en casa.

A partir de este pilotaje en diversos estados del país, el Programa Nacional de Tamizaje del Cáncer Cervicouterino modificó su estrategia de detección introduciendo la prueba de ADN de VPH en combinación con el tamizaje a través de la citología cervical, en la población de mujeres en edad de riesgo (25 a 64 años), y la aplicación de la vacuna en la población infantil femenina ${ }^{(19)}$.

\section{APARTADO METODOLÓGICO}

El trabajo etnográfico que da origen a este artículo se realizó en la clínica de displasia en una ciudad del estado de Veracruz, ubicada en un centro especializado en oncología. Tuve la oportunidad de permanecer diariamente durante periodos mensuales en la clínica -seis a lo largo de tres años- observar el proceso de atención que se realizaba y entrevistar al personal de salud que brindaba sus servicios a mujeres enviadas para la detección y atención de este tipo de cáncer. Realicé entrevistas a las tres médicas colposcopiastas de la clínica de displasia, a las tres enfermeras y la trabajadora social asignadas al servicio y a una médica de primer nivel de atención. Las entrevistas en profundidad las realicé en diferentes contextos: en la clínica, después de la consulta, o bien en los consultorios privados de las médicas. La duración de las entrevistas fue variable, en promedio de 120 minutos, todas las entrevistas fueron grabadas con la autorización de las participantes y posteriormente trascritas. Realice una codificación de la información obtenida, basada en las categorías y variables del proyecto y un análisis de contenido con base en la matriz de información.

La observación participante se realizó en los diferentes espacios de atención de la clínica: durante la consulta, en la sala de espera y en el área de recuperación. Elaboré una guía de observación para cada uno de los espacios y registré en el diario de campo todos los sucesos que se presentaban, esta información también fue codificada sobre la base de una serie de categorías, y analizada.

En la clínica laboran tres médicas colposcopistas, personal de enfermería y trabajo social, durante el desarrollo del trabajo se obtuvo el consentimiento informado del personal de la clínica de displasias, así como de personal de primer nivel de atención, se utilizaron seudónimos para cada informante con el fin de preservar la identidad de cada una de ellas, y se les informó de nuestro compromiso de guardar su identidad y su derecho de conocer la información que estábamos obteniendo, en el momento que lo solicitaran. El proyecto fue evaluado y autorizado por el grupo de personas que integraban el Comité de Ética del hospital, aunque durante la época de nuestro trabajo no se encontraba conformado oficialmente.

\section{UNA CLÍNICA DE DISPLASIAS EN VERACRUZ}

Esta unidad de segundo nivel de atención está vinculada con las unidades médicas de primer nivel a través de las referencias que realizan los médicos ante un diagnóstico presuntivo de displasia, infección por virus papiloma humano o cáncer cervicouterino y se vincula con el tercer nivel de atención, con el que comparten el espacio físico, al referir a aquellas mujeres con diagnóstico de cáncer invasor o metástasis que requieren la intervención de especialistas en oncología clínica o quirúrgica.

Una vez descrito someramente el panorama epidemiológico de este tipo de cáncer en México y en el estado de Veracruz, nos interesa cuestionarnos: cuál es la opinión de los actores sociales que implementan el programa de detección en cáncer cervicouterino en el estado y cuáles han sido sus limitaciones, problemas y resultados, por lo que daremos voz a estos sujetos para que den cuenta de esta historia: 
Hace 25 años, aunque existía un programa de detección, realmente las estrategias que se realizaban eran mínimas, no se invitaba a las mujeres a que se hicieran el pap [Papanicolaou]. Veracruz estaba entre los primeros lugares de mortalidad por esta causa, no se realizaban campañas, ni se lograban las metas. En el año 2000 se hizo una evaluación del programa y a partir de ahí se implementan nuevas estrategias: se capacitó al personal en la toma adecuada de la muestra, se organizó una capacitación intensiva con los citotecnólogos sobre la lectura adecuada de las muestras de citología y se puso gran énfasis en el seguimiento de las mujeres que resultaban positivas. En esa época se creó la primera clínica de displasia en el estado, la cual se instaló en el Centro Estatal de Cancerología, con resistencia por parte del personal directivo y operativo del hospital (cirujanos oncólogos y ginecólogos). Hacía ya algunos meses que había llegado un colposcopio al hospital, pero se encontraba en el consultorio privado del director y no tenía la menor intención de proporcionarlo para el programa. Se capacitó como colposcopistas a personal médico en el Hospital General de México, que fue el pionero en la formación de colposcopistas en el país. Se inició un proceso de promoción y difusión del programa con el apoyo de una organización extranjera (japonesa) que realizó de forma externa una evaluación del programa estatal, proporcionando financiamiento para la capacitación del personal, la dotación de tecnología y la organización de reuniones anuales del personal involucrado, teniendo mayor apoyo en el caso de los colposcopistas. Este impulso ocasionó una disminución en la tasa de mortalidad por esta causa en nuestro estado. (Sonia, médica colposcopista)

Se estaban impulsando las clínicas de displasia a nivel nacional, porque se había demostrado que existían otras formas de detectar y atender el problema del cáncer en las mujeres, antes lo que se hacía era hacer el pap y en caso de reportarse alteraciones se biopsiaba [toma de una biopsia del cuello de la matriz] sin ver, en forma horaria, y después si salía positiva se hacía una histerectomía, entonces no había más que dos pasos: un pap y la histerectomía, entonces se empezó a difundir que había otra forma de diagnóstico a través del colposcopio que es un microscopio para poder ver a mayor tamaño (Elisa, médica colposcopista)

Estas médicas han vivido los cambios en la implementación de una estrategia de salud, a través de este programa nacional, han sido actoras centrales de las transformaciones, al operar el programa previo a la utilización de una herramienta como el colposcopio, capacitarse después en el Hospital General de México y echar a andar el programa en la clínica de displasia, dando cuenta del impacto en el desarrollo de la detección, las decisiones y el manejo médico.

Son los actores sociales quienes ponen en acción las políticas públicas en salud, a través de operar un programa específico, en este caso el programa de detección oportuna de cáncer cervicouterino. El sujeto social involucrado es el personal de salud que se ubica en los tres niveles de atención. En el primer nivel se realiza el tamizaje -prueba de rastreo en la población objetivo para detectar los casos sospechosos- para sacar a la luz entre muchas mujeres aquellas que puedan tener problemas y que deben pasar al segundo nivel en la clínica de displasia, donde hay que descartar la enfermedad o visualizarla y tratarla si es posible y, si no, enviarlas a tercer nivel con los oncólogos.

Durante mi trabajo de campo tuve oportunidad de entrar en contacto con la médica de un centro de salud de la zona de influencia de la clínica de displasia, ella nos habló de la forma en que se desarrolla el programa en su centro de atención:

Nosotros siempre trabajamos con metas, el pilar del programa es enfermería. Porque aquí hay mucha consulta, entonces 
ellas [las enfermeras] nos apoyan el cien por ciento en la toma de muestras de pap, cuando ven en la paciente algún dato anormal, ellas nos Ilaman, si tienen flujo o algo nos hablan, entonces al médico que le corresponda va a ver a su paciente y ya le indica medicamento. Tenemos una población de 17.586, la meta en edad fértil es de 5.033, esa es la cobertura, es a quienes tenemos que tener en control de planificación familiar y en consecuencia la toma de Papanicolaou. La cobertura es del $21 \%$ en este trimestre. (Alba, médica del primer nivel de atención)

Como podemos corroborar en este testimonio la cobertura está muy por debajo de las metas asignadas a estas unidades médicas por la normatividad institucional, pero cuáles son las razones por las que esto sucede. La estrategia central en el primer nivel de atención entre población vulnerable está basada en el programa Oportunidades (hoy nombrado Progresa) principal estrategia del gobierno federal para contrarrestar la pobreza del país.

A través de las familias de Oportunidades tenemos mucho contacto. Hay tres talleres de mujeres, dos con información y en el tercero se toma pap y detección clínica de mama. Así logramos hacer muchas detecciones, hay algunas mujeres que nunca se lo había hecho y ahí se lo hacemos. Porque si les decimos que tal día vengan, no vienen, en cambio a los talleres tienen que venir a la fuerza. Así lo hacemos cada año, para poder darle continuidad. A los médicos no les gusta mucho ir a los talleres, pero les sirve para distraerse de lo de todos los días, de tanta consulta, para despejarse y relajarse de la consulta. (Alba, médica del primer nivel de atención)

Si bien los talleres a los que hace referencia la médica son la estrategia de educación para la salud que el programa de detección considera centrales, al parecer se realizan sin convicción y compromiso del personal de salud, sino como una rutina, se convierten en una estrategia de captación de la población para poder cubrir la metas, entrando en juego la falta de sensibilidad y capacitación técnica del personal para el trabajo de campo en combinación con limitaciones culturales en la relación con grupos sociales de diferente estrato social y etnia, y para la población es la forma en que aseguran obtener los beneficios del programa de apoyo económico para los escolares y mujeres jefas de familia.

Qué representaciones tienen las médicas de segundo nivel en la clínica de displasia, quienes reciben a las pacientes referidas de los centros de salud, sobre la forma en que la estrategia de detección se realiza en campo:

No comprendo por qué en los centros de salud no se hace un barrido buscando a las mujeres que no se han hecho el pap, son mujeres que van a los centros de salud, pero no se les pregunta ni se les convence para que se los hagan, incluso tuve una paciente diabética que cada mes estaba en el centro para el control metabólico, pero su médico nunca le preguntó, ni la convencieron de hacerse la detección y ahora viene con un NIC 2. (Elisa, médica colposcopista)

Los resultados de las pruebas de $\mathrm{Pa}$ panicolaou se reportan sobre la base de la clasificación de neoplasias intraepitelial cervicouterina grado 1, 2 y 3, dependiendo de su gravedad: NIC 1 displasia leve, NIC 2 displasia moderada y NIC 3 displasia grave.

En las comunidades se hace la promoción con las mujeres de Oportunidades, pero las que no tienen ese beneficio no se les tome en cuenta, así puede ser que una mujer esté enfrente de la otra, una se haya hecho el pap y la otra no. Pero es que el programa de Oportunidades tiene muchas irregularidades, me han dicho que se hacen las visitas a las comunidades y se saltan ciertas casas porque como son gente que los hijos trabajan fuera y mandan dinero, ya no se incluyen en el programa, aunque sean campesinos. (Graciela, médica colposcopista) 
Cabe preguntarse por qué si al parecer hay información proporcionada por las instituciones y en los medios de comunicación, no se ve reflejada en un incremento de las detecciones a tiempo. Al parecer, la información no tiene eco ni en las mujeres, ni en parte del personal de salud. La decisión que lleva o no a las mujeres a realizarse la prueba de tamizaje son muy complejas y aunque en este texto no fue el centro de interés mostrar las representaciones de las usuarias del programa, si es importante hacer evidentes las condiciones institucionales y las interpretaciones que de ello tiene le personal de salud. Siendo importante señalar que una de las características de la biomedicina es poner el acento en la atención, en lo curativo, secundarizando la prevención, si bien en el discurso institucional y estatal se dice que lo mejor es prevenir en la operación se hace evidente un demérito de lo preventivo, siendo relegado al profesional de la salud con menor jerarquía que el médico.

Las mujeres dicen que no saben cuándo deben hacerse el pap, la mayoría se lo hicieron después de varios años de vida sexual activa. (Elisa, médica colposcopista)

...la razón es que es muy miedosa, le daba mucho miedo que le fueran a decir que tenía algo, por eso prefería no hacérselo [el pap], pero ahora con el programa Oportunidades se lo tenía que hacer a fuerza. (Julia, enfermera)

Lo que la gente dice es que duele, pero las enfermeras tratan de convencerlas. Otras también dicen que como ya no tienen esposo, para que se lo van a hacer, pero yo les digo que no, aunque no tengan marido se tienen que revisar. (Alba, médica del primer nivel de atención)

También se ven señoras que nunca se han hecho un pap en su vida y no es porque no sepan, porque si han visto en la tele y en el centro de salud que tiene que hacerse eso para cuidarse, pero no aceptan porque sus esposos no les dan permiso, porque no quieren que nadie vea a sus mujeres, nada más ellos hacen uso de eso, pero nadie más. Aunque sean enfermeras mujeres quienes le hacen el pap, ellos no quieren y las señoras los obedecen, pero ha de ser porque son mujeres que no se quieren, por eso no cuidan su cuerpo. (Claudia, trabajadora social)

Son muchos los factores que influyen para que una mujer tome la decisión de realizarse una detección como el Papanicolaou: disponibilidad del servicio, diversos tipos de accesibilidad, siendo muy importante la accesibilidad cultural, las relaciones de género, los saberes respecto de la enfermedad y la toma de la detección, entre otros. Lo que nos abre una infinidad de ventanas de oportunidad en el análisis cualitativo de la información de las usuarias, aunque en este momento no exploraremos sobre ello.

La opinión del personal de salud es que hoy el programa en el estado está nuevamente desprotegido, desde el punto de vista financiero, no se cuenta con el personal suficiente y capacitado, principalmente en el área de la toma de la muestra, que es donde se inicia el proceso:

...son las auxiliares de enfermería las que realizan la toma en los centros de salud, sobre todo en el área rural, sin contar con los recursos materiales ni técnicos para que la muestra sea de calidad, los médicos no se involucran en acciones de prevención. (Sonia, médica colposcopista).

En los centros de salud, el programa lo llevan las enfermeras y muchas no están capacitadas, hacen tomas por fuera de la zona adecuada, en ocasiones deberían tratar de buscar la lesión en el canal [del cuello de la matriz], deberían hacer la toma con citobrux [cepillo especial para la toma dentro del canal del cuello de la matriz] pero incluso a veces no se puede tomar en mujeres con un cervix pequeño y atrófico, su zona de trasformación [cervix atrófico pequeño y 
cerrado que se observa en mujeres posmenopáusicas] se ha perdido, los médicos de primer nivel no saben eso, por eso no hacen un esfuerzo por hacer mejores detecciones, ellos no están capacitados en el programa. Incluso se ha visto que algunos médicos no saben lo que es una displasia o no tienen información sobre la IVPH. (Elisa, médica colposcopista)

Existen limitaciones de diverso tipo, capacitación y recursos en los servicios de salud que se expresan en todos los niveles de atención impactando el desarrollo del programa:

Una paciente con diagnóstico de NIC 1 le comenta a la médica sobre un familiar suyo, que están viendo en Actopan con un médico particular, porque el centro de salud que le toca no trabaja bien, nunca tienen espejos o les hace falta la laminilla, por eso ella decidió mejor ir con una particular ahí la doctora le está poniendo yodo para revisarla. [Notas de campo]

Además, se producen sobrecargas en las actividades del personal de salud que ocasiona errores, desgaste e impacta en la relación terapéutica:

Las enfermeras son las que tienen éxito en la toma del pap, porque se comprometen con el programa, aunque tengan mucho trabajo hacen las tomas, aunque a veces la lámpara de chicote [instrumental indispensable para alumbrar la zona genital de la mujer y tomar una muestra adecuada] no sirve. Tratan de evitar no regresar a las pacientes. Son muy cooperativas en ese sentido. Porque los médicos tienen mucha consulta, incluso se tiene que regresar a la gente porque no tenemos ya posibilidades de verlos a todos. La institución quiere que con el mismo personal y con pocos recursos se saque todos los programas. Hay veces que tenemos mucho trabajo y tenemos mucho estrés, porque es mucho el trabajo. (Alba, médica del primer nivel de atención)
Una situación que se reconoce como problemática en la consecución del programa de detección es la imposibilidad de otorgar la cita oportuna a la gran cantidad de pacientes de primera vez que cada semana llegan a la clínica de displasias, enviadas por la jurisdicción sanitaria. Para las que hay que otorgarles atención inicial, el procedimiento es el siguiente: las citologías tomadas en los centros de salud se analizan y diagnostican en los laboratorios de citología del hospital y los resultados son recopilados por la jurisdicción sanitaria, los registran y envían al centro de salud correspondiente, aquellos resultados que son positivos a lesión intraepitelial de cualquier grado (NIC) o IVPH, se envían a la clínica de colposcopia para que les otorguen una cita. La normatividad indica que debe proporcionarse cita oportuna, hay que dar prioridad a las pacientes de primera vez en su atención. Si el resultado es de alto riesgo se les da una cita a corto plazo, pero si el resultado es de bajo riesgo, se programan las citas a dos y tres meses, debido a la gran cantidad de pacientes subsecuentes que tienen que atender las médicas. La institución les pide a las médicas que generen una estrategia para poder otorgar más citas de primera vez y reducir las subsecuentes. Lo que la doctora Elisa propone es:

...dar de alta la mayor cantidad de pacientes y darles su cita subsecuente más espaciada. A una paciente de primera vez, que se le de todo el tiempo necesario [50 a 60 minutos] porque ahí es donde vas a explicar todo lo de la enfermedad y sus cuidados, pero después ya no, no podemos estar perdiendo el tiempo explicando otra vez lo mismo, cuando la paciente no entiende, es porque no entiende y ya, cada quien debe hacerse responsable. (Elisa, médica colposcopista)

...en la segunda y tercera cita es cuando se les da el resultado de la biopsia o cuando se les hace el Asa [electrocirugía terapéutica, realizada en el cuello de la matriz], también tienes que explicarles, se tarda tiempo en comprometerlas para 
que evolucionen bien. (Sonia, médica colposcopista)

...ya no, ahí ya no es necesario, ya se dijo todo en la primera ocasión, porque si no, nunca terminas y no hay tiempo para eso. Tú lo que debes hacer como colposcopista es dar tu diagnóstico y tratamiento colposcopico, no tienes por qué estarte metiendo en otra cosa. Tu compromiso y responsabilidad está de la cortina de tu consultorio para acá, lo que pase afuera o allá en el otro lado no te corresponde, no es tu responsabilidad. Si ellas no hacen cambios en su vida, ya no es responsabilidad del médico. Nosotras como colposcopistas damos indicaciones de los cambios necesarios para su mejoría, pero si las pacientes tienen piso de tierra en su casa, su marido las golpea o ellas no pueden comer verdura porque en su pueblo eso no hay o no les gusta, eso ya es responsabilidad de ellas, porque no puedes cambiarles su vida, a nosotras lo que nos toca es la lesión, no podemos resolver la pobreza. (Elisa, médica colposcopista)

Este discurso de la médica nos permite reflexionar sobre la perspectiva biologicista de la práctica biomédica, que concentra su energía en las acciones sobre el cuerpo, con un enfoque anatómico estructural, para este caso el cérvix de la mujer enferma, pero pierde de vista y/o no considera útil o posible incidir en las condiciones de vida de estas mujeres y en sus interacciones sociales. El saber médico se construye a partir de los modelos explicativos de los diferentes actores sociales, poniendo en juego sus interpretaciones respecto de la salud, de la enfermedad, en este caso de una enfermedad de transmisión sexual, en el que se expresan posturas que acercan o distancian culturalmente a los actores, al elaborar modelos explicativos diferentes, lo que incluso en ocasiones genere que la autoridad médica culpabilice a la paciente de su situación de enfermedad y desatención.

Otra evidente inconsistencia y grave obstáculo en el desarrollo del programa es la elevada proporción de mujeres que no acude a la cita en la clínica de displasia. No obstante, la saturación en el número de pacientes que estas médicas tienen que atender diariamente y el consecuente diferimiento de las citas otorgadas, existe una elevada proporción de mujeres que no acuden a su cita y en consecuencia pierden la oportunidad de ser valoradas por esta instancia especializada. Al preguntar por qué las mujeres no llegan a su cita de primera vez, la contestación es:

...una vez otorgada la cita en la clínica de displasia se informa a los centros de salud para que localicen a las mujeres. Desgraciadamente, en muchas ocasiones este proceso se hace cercano a la fecha de la cita, lo que ocasiona inconvenientes para poder lograr que las mujeres vayan. (Alba, médica del primer nivel de atención)

Se avisa a las mujeres muchas veces un día antes de la cita o días después, existen problemas para localizar a la mujer o ellas no pueden reunir las condiciones necesarias para acudir a su cita, de un día para otro, o bien la cita ya se perdió, eso es lo más frecuente. Entonces la gente prefiere ir al médico privado, pagar porque tienen miedo de la enfermedad, aunque no tienen recursos económicos prefieren endrogarse para pagar el particular, pero en su comunidad y rápido. (José, enfermero)

La médica del primer nivel de atención nos refiere:

...hay ocasiones en que no se pueden localizar [a las mujeres] porque ya no viven aquí, lo que pasa es que aquí la gente renta, mucha gente no es de aquí, sino que rentan, entonces se cambian frecuentemente de domicilio y no puede uno localizarlos, es gente que viene de los ranchos a vivir aquí o vienen de muy lejos y se vuelven a ir. [...] Cuando se entrega el pap, ya no se revisa a la paciente porque no hay tiempo, nos 
entregan el resultado para el otro día o nos hablan por teléfono diciendo que hay tal resultado y la cita es para tal día, hay que localizar a la mujer y mandarla a tiempo para que no pierda su cita. Quién sabe por qué no se avisa a tiempo de la jurisdicción, si la cita es de un día a otro y no se localiza, ya no podemos hacer nada. (Cele, enfermera)

Durante una reunión de evaluación del desempeño entre las médicas de la clínica de displasia y las responsables del Programa Nacional de Tamizaje del Cáncer Cervicouterino a nivel jurisdiccional, se señala de forma central que:

\begin{abstract}
...aproximadamente, el $40 \%$ de las mujeres que son enviadas por citologías anormales no llegan a su cita, no se presentan. Por lo que es necesario generar estrategias para que estas mujeres lleguen, ya que, si bien la mayoría son NIC1, se sabe que entre estas puede haber mujeres que después de la colposcopía se le hagan diagnósticos más graves y si no llegan a sus citas no serán atendidas. [Notas de campo]
\end{abstract}

La doctora Sonia insiste en que, si no se tiene contacto y retroalimentación con el primer nivel, las pacientes van a seguir faltando a las citas o llegando sin exploración física y sin tratamiento para procesos infecciosos que impiden que se tome la biopsia, generando una cita más. Sin información y sensibilización del personal del primer nivel, el programa no puede continuar, es necesario hacer una reunión con médicos del primer nivel, capacitarlos, comunicarse con ellos para preguntarles qué pasa. La doctora Elisa opina que eso no le corresponde al segundo nivel, sino que hay que darle información a la jurisdicción y ellos tienen que tomar las acciones.

Es evidente una burocratización del sistema de salud, división del trabajo y falta de responsabilidad y comunicación con la paciente. Junto a la falta de coordinación entre los diferentes niveles de atención, coexiste un desconocimiento por parte de cada una de las instancias, del proceso, del programa, de las necesidades, condiciones, requerimientos y dificultades que enfrentan los actores que desarrollan las acciones en cada uno de los niveles, lo que repercute indiscutiblemente en el tipo de proceso que se realiza y que impacta, sobre todo, en las más vulnerables que son las mujeres, que pueden estar cursando un problema de salud que, de no ser atendido, puede desencadenar en un cáncer y llevarlas a la muerte.

Un actor excluido del programa de detección es el varón, compañero sexual de las mujeres que enferman de cáncer cervicouterino. Debemos cuestionarnos cuáles son las razones por las que los varones se excluyen de las acciones de detección y manejo de esta patología, aun cuando se reconoce su participación como una condición para la presencia de la enfermedad:

Sería bueno trabajar sobre todo con las parejas sexuales, porque aquí hay mucha gente que sale a trabajar, emigran a México o gente de los ranchos que viene a trabajar aquí, por eso que tenemos resultados con displasia. La mayoría de los esposos están en México o en EE.UU., o se van a trabajar afuera y regresan los fines de semana, esa es una razón que sea una zona de riesgo, porque esos esposos tienen otras parejas sexuales. (Alba, médica del primer nivel de atención)

Yo creo que a los hombres hay que darles información, decirles lo que está sucediendo porque ellos no son ni tontos ni insensibles, ellos son capaces de hacerse responsables de lo que hacen si están informados, cuando hemos hecho eso ha funcionado porque las mujeres mejoran. Pero en el hospital eso no es fácil porque no nos damos abasto ni con lo que tenemos que hacer con las mujeres, no hay quien se haga cargo de los varones, además debe ser alguien que tenga empatía con ellos, nosotras no. Pero la mejor forma de tratar a los hombres es hacer que se pongan el condón, porque si ellos no quieren cambiar sus hábitos lo 
que tenemos que hacer es proteger a las mujeres. (Sonia, médica colposcopista)

Ni los hombres ni las mujeres que nosotros vemos están informados de nada, ellos no saben del virus, ni que causa enfermedad, ni que la forma en que viven los va a llevar a estar enfermos, cuando llegan a la clínica llegan porque los mandaron, no porque ellos sepan algo y además muchas veces no entienden por qué les decimos las cosas, si para ellos eso no es para enfermarse. (Elisa, médica colposcopista)

A los esposos no se les hace nada aquí, hubo un tiempo en que sí, había un doctor que revisaba a los esposos, algunos sí venían, otros no querían venir, les hacía tratamiento y se preocupaban porque sus mujeres se enfermaban, querían ayudar, se ponían el condón y ya no andaban con otras mujeres, pero a otros no les importaba, no venían a los tratamientos y no usaban el condón, entonces sus mujeres seguían enfermas. Yo no creo que eso les ayude a las mujeres, además aquí no se puede atender a toda la gente, imagínese las doctoras no se dan abasto con tanto trabajo. (Julia, enfermera de clínica de displasia)

La exclusión de uno de los actores involucrados en este tipo de enfermedad se encuentra íntimamente relacionada con saturación de los servicios ante la falta de personal técnico, pero también con relaciones de género que influyen directamente en el quehacer profesional de estas médicas y con una perspectiva enfocada en la enfermedad (localizada en la mujer) perdiendo de vista un enfoque de promoción y educación para la salud que voltearía la mirada a la pareja sexual.

\section{DISCUSIÓN}

Los trabajos sobre la salud de las mujeres $y$, especialmente, sobre la presencia e impacto del cáncer en esta población ha sido abordado desde diferentes disciplinas, existen diversidad de trabajos en América Latina interesados en las representaciones sociales de las mujeres y cómo influyen en la detección y en el desarrollo de la enfermedad ${ }^{(20,21,22)}$. El interés en los actores que operan los programas surge, principalmente, en el área de políticas públicas y servicios de salud que se enfocan en la evaluación de los programas y en trabajos de salud colectiva que ponen el acento en una mirada crítica respecto de la perspectiva de la prevención en salud, considerándola una práctica política y social, que rebasa la perspectiva individualista, una acción crítica y reflexiva sobre la vida al tomar conciencia de la propia existencia, la capacidad de actuar con autonomía y un modo de ejercer el derecho a la salud(23); postura que compartimos y consideramos necesario retomar en los diversos trabajos sobre el proceso salud-enfermedad que se enfocan en la prevención.

Este acercamiento a la operación de un programa tan importante como es el Programa de Detección Oportuna del Cáncer Cervicouterino pone en evidencia la urgencia de reconocer al cáncer cervicouterino como una enfermedad multifactorial, no es solo la IVPH y su prevención el eje que debe guiar la intervención preventivo-terapéutica. Realmente es importante poner el énfasis en la educación y la promoción de la salud, pero no solo entre la población sino también entre el personal de salud, generando un compromiso real para incidir en una detección temprana de la enfermedad en la población más vulnerable. Para lo cual es indispensable contar con personal sensible y capaz de realizar su práctica con pertinencia cultural, solo conociendo los modelos explicativos de los diferentes actores será posible tratar de establecer puentes entre ellos.

El saber que construyen los prestadores de servicio está conformado bajo condiciones de clase, étnicas y de género que determinan la forma en que dichos sujetos sociales interpretan la enfermedad que las mujeres padecen, pero no solo de forma técnica, sino impregnada de prenociones sobre cómo adquirieron la enfermedad, cómo se atienden, cómo viven 
con ella, estas representaciones determinan prácticas distantes en la atención e incluso llegan a culparlas de su condición de enfermas.

Sus representaciones sociales y sus prácticas respecto de la enfermedad y del programa de detección nos muestran una constante combinación y síntesis de perspectivas teóricas biomédicas que interactúan con condiciones sociales, políticas y económicas en las que se da su práctica, que permiten u obstaculizan la operación de una técnica diagnóstica, preventiva o terapéutica y que, a la vez, se articulan con sus representaciones respecto del tipo de mujeres con las que interactúan, construyéndose así su saber profesional.

El saber médico de estos prestadores de servicios expresa diversas características del modelo biomédico que centra su explicación en el biologicismo, perdiendo de vista el contexto histórico social que le da sentido, privilegiando su acción diagnóstico-terapéutica sobre la prevención-educación, expresando una relación asimétrica con las pacientes y una práctica burocratizada en un contexto institucional que promueve el individualismo, la división de las responsabilidades y la lejanía con las pacientes.

Es también de gran importancia reconocer las condiciones estructurales y las relaciones desiguales en que viven las poblaciones en riesgo y que determinan que las mujeres veracruzanas más pobres sigan muriendo por esta enfermedad, que es definitivamente prevenible y curable en sus etapas tempranas. Si bien es prioritario el compromiso real del Estado para generar las condiciones óptimas para el desarrollo del programa y la intervención de las poblaciones y los sujetos sociales en la sensibilización y gestión de procesos de detección y atención desde los propios conjuntos sociales. esto no exime la responsabilidad social y ética de las instituciones de salud para brindar servicios eficaces al contar con los recursos materiales y humanos que realicen las acciones del programa de detección, que en el papel puede ser muy convincente pero en la práctica real muestra infinidad de errores y obstáculos.

Creemos que hay que generar las condiciones para que las mujeres se apropien del cuidado de su salud, promoviendo acciones de autogestión y reivindicación de los derechos, que parten de contar con las condiciones de vida digna que permitan transformar las representaciones sobre el autocuidado y bienestar, como parte del derecho a la salud.

\section{REFERENCIAS BIBLIOGRÁFICAS}

1. Subsecretaría de Prevención y Promoción a la Salud. Programa de acción específico 2007-2012 cáncer cervicouterino. México: Secretaría de Salud; 2008.

2. Sánchez-Barriga JJ. Mortalidad por cáncer cervicouterino. Gaceta Médica de México. 2012; 148:42-51.

3. Hernández-Hernández DM, Apresa-García T, Patlán-Pérez RM. Panorama epidemiológico del cáncer cervicouterino. Revista Médica del IMSS. 2015;53(Supl 2):S154-S161.

4. Subsecretaría de Prevención y Promoción a la Salud. Programa de acción específico: Prevención y control del cáncer de la mujer 2013-2018. México: Programa Sectorial de Salud; 2016.
5. Torres-Poveda KJ, Arredondo-López AA, DuarteGómez MB, Madrid-Marina V. La mujer indígena, vulnerable a cáncer cervicouterino: Perspectiva desde modelos conceptuales de salud pública. Salud en Tabasco. 2008;14(3):807-815.

6. Drain P, Colmes K, Highes J. Determinants of cervical cancer rates in developing countries. International Journal of Cancer. 2002;100(2):199-205.

7. Organización Mundial de la Salud. Prevención y control integrales del cáncer cervicouterino: un futuro más saludable para niñas y mujeres. Washington DC: OMS; 2013.

8. Montes E. Control de calidad en los laboratorios involucrados en los programas de detección del cáncer cervicouterino en Cáncer cervicouterino: Diagnóstico, prevención y control. 2a ed. México: Editorial Panamericana, UNAM, INSP; 2005. 
9. Dirección General de Información en Salud (DGIS). Cubo de defunciones [base de datos en Internet]. México: SINAIS, Secretaría de Salud; 2012 [citado 12 ago 2016]. Disponible en: https://goo. $\mathrm{gl} / 4 \mathrm{zj}$ Pts

10. Organización Panamericana de la Salud. Control integral del cáncer cervicouterino: guía de prácticas esenciales. 2a ed. Washington DC: OPS; 2016.

11. Hernández $\mathrm{P}$, Lazcano $\mathrm{EC}$, Alonso $\mathrm{P}, \mathrm{Cruz}$ A, Meneses F, Hernández M. Análisis costo beneficio del Programa de Detección Oportuna del Cáncer Cervicouterino. Salud Pública de México. 1997;39(4):379-387.

12. Estados Unidos Mexicanos, Secretaría de Salud. Norma oficial mexicana NOM-014-SSA2-1994, para la prevención, detección, diagnóstico, tratamiento, control y vigilancia epidemiológica del cáncer cervicouterino [Internet]. 1998 [citado 10 mar 2016]. Disponible en: https://goo.gl/XmjdHo.

13. American Cancer Society. Cancer Facts \& Figures 2014 [Internet]. Atlanta: American Cancer Society; 2014 [citado 10 mar 2016]. Disponible en: https://goo.gl/KJW7SN.

14. Palacio-Mejía LS, Lazcano-Ponce E, AllenLeigh B, Hernández-Ávila M. Diferencias regionales en la mortalidad por cáncer de mama y cérvix en México entre 1979 y 2006. Salud Pública de México. 2009;51(Supl 2):208-219.

15. Torres-Mejía G, Ortega-Olvera C, ÁngelesLlerenas A, Villalobos-Hernández AL, SalmerónCastro J, Lazcano-Ponce E, Hernández-Ávila M. Patrones de utilización de programas de prevención y diagnóstico temprano de cáncer en la mujer. Salud Pública de México. 2013;55(Supl 2):241-248.
16. World Health Organization. National Cancer Control Programmes: Policies and managerial guidelines. 2a ed. Geneva: WHO; 2002.

17. Gutiérrez-Delgado C, Báez-Mendoza C, González-Pier E, Prieto de la Rosa A, Witlen R. Relación costo-efectividad de las intervenciones preventivas contra el cáncer cervical en mujeres mexicanas. Salud Pública de México. 2008;50(2):107-118.

18. Secretaria de Salud. Lineamientos para la semana de sensibilización del cáncer de cuello uterino. México: Dirección Cáncer de la Mujer; 2015.

19. Guevara Herrera M. Prevención integral del cáncer cervicouterino en municipios con alta marginación. México: Secretaría de Salud Veracruz; 2015.

20. Tamayo Acevedo L, Chávez Méndez MG, Henao Franco L. Cáncer de cuello uterino: más allá de lo que es la percepción de las mujeres de Antioquia (Colombia) y Colima (México), 2008. Estudios sobre las Culturas Contemporáneas. 2010;(32):41-61.

21. Lamadrid, S. Aspectos socio-culturales de la sexualidad como factores obstaculizantes de la prevención secundaria del cáncer cérvico uterino. Cadernos de Saúde Pública. 1998;14(Supl. 1):33-40.

22. Pimentel AV, Panobianco MS, Almeida AM, Oliveira ISB. Percepción de vulnerabilidad entre mujeres con diagnóstico avanzado de cuello del útero. Texto \& Contexto - Enfermagem. 2011;20(2):255-262.

23. Ponce M. La prevención del cáncer del cuello de útero y de mama en servicios de salud y organizaciones no gubernamentales de la Ciudad Autónoma de Buenos Aires. Salud Colectiva. 2013;9(2):215-233. 